Methods The study was carried out in Federal Medical Center, in South East Nigeria. Questionnaire and checklist was used to assess hand washing practice among 240 care providers and hand washing facilities in clinics and wards.

Result The health workers surveyed included nurses (54.0\%), interns $(19.0 \%)$, resident doctors $(12.7 \%)$ and medical officers (9.5\%). Majority of them (96.8\%) knew the importance of handwashing in the prevention of hospital-acquired infections. More than $50 \%$ had good knowledge of hand hygiene. About $54 \%$ of them will always wash their hands between direct contact with patients, and $68 \%$ after an invasive procedure, $92 \%$ when hands are visibly soiled, $67 \%$ after removal of gloves and $77 \%$ after personal body functions. Most common barriers to hand hygiene practice identified included, unavailability of water $(61.9 \%)$ and alcohol-based agent $(69.8 \%)$. About $14 \%$ of them did not wash their hands when an opportunity to do so presented. Among those that washed their hands the mean duration of hand washing was 39.6 $\pm 24.4 \mathrm{~s}$. Facility assessment showed that all the wards and clinics had designated place for hand washing. Only one clinic had alcohol cleaning agent and running water, $50 \%$ soap, $83.3 \%$ liquid detergent, $75 \%$ re-usable towel and $91.7 \%$ water storage container.

Conclusion The study supports earlier findings that hand hygiene practice in developing countries is relatively low and lack of supplies are barriers to its practice.

\section{SP6-61 RISK ASSESSMENT OF OCCUPATIONAL ACCIDENTS AND PSYCHOLOGICAL STATUS AMONG EMPLOYEES IN THE MILITARY INDUSTRY IN NORTHWEST RUSSIA}

doi:10.1136/jech.2011.142976q.32

\begin{abstract}
${ }^{1,2} \mathrm{Z}$ Varakina, ${ }^{*} \mathrm{~A}$ Vyazmin, ${ }^{2} \mathrm{~A}$ Sannikov, ${ }^{3,4} \mathrm{~A}$ Grjibovski. ${ }^{1}$ Department of Research Management, Northern State Medical University, Arkhangelsk, Russia; ${ }^{2}$ Department of Public Health, Health Care and Social Work, Northern State Medical University, Arkhangelsk, Russia; International School of Public Health, Northern State Medical University, Arkhangelsk, Russia; ${ }^{4}$ Norwegian Institute of Public Health, Oslo, Norway
\end{abstract}

Introduction Occupational health and psychological comfort at work is important part of occupational safety promotion and reflects quality of life of employees. The aim of the study is to perform qualitative risk assessment of accidents and to study psychological status of employees in military industry in Northwest Russia.

Methods A cross-sectional questionnaire-based study was conducted in one of the military enterprises in the city of Arkhangelsk in 2010 in a random sample of employees $(n=331)$ who reported on lifestyle, work experience and psychological comfort at work. Occupational hazards were analysed by criteria for risk assessment of accidents at working place (Tampere, 1994). Proportions were analysed by $\chi^{2}$ tests.

Results Most of the responders were males $(70.1 \%, n=232)$ aged 29.6 years on average. There were no differences by gender in psychological comfort at working place $(p=0.76)$. Only $16.9 \%$ of the responders estimated psychological comfort at the high level. Altogether, $71.0 \%$ responders considered their working place as dangerous $(n=235)$. Among males this proportion was higher than among females $(81.9 \%$ vs $45.5 \%, \mathrm{p}<0.001)$. Substantial and intolerable risks of accidents were found in $14.2 \%$ and $11.8 \%$ of the responders, respectively. Substantial risk was estimated in $18.1 \%$ of males and $5.1 \%$ of females $(p<0.001)$. Corresponding proportions for intolerable risk were $13.8 \%$ vs $7.1 \%$ ( $p<0.001)$.

Conclusions Employees in Russian military industry have high risks of occupational accidents in combination with absence of psychological comfort at working place.

\section{SP6-62 "PRECISE-A MODEL OF COMMUNITY PARTICIPATION IN CHILD INJURY PREVENTION IN BANGLADESH, IMPLICATION FOR OTHER LOW INCOME COUNTRY"}

doi:10.1136/jech.2011.142976q.33

${ }^{1,2} \mathrm{~K}$ Ul Baset, ${ }^{*} \mathrm{~S}$ M Saidur Rahman, ${ }^{1} \mathrm{~A}$ Rahman, ${ }^{1} \mathrm{~A}$ K M Fazlur Rahman, ${ }^{2} \mathrm{E}$ Towner. ${ }^{1}$ Centre for Injury Prevention and Research Bangladesh (CIPRB), Dhaka, Bangladesh, ${ }^{2}$ University of the West of England, Bristol, UK

Introduction Community participation is an important component of a community based health intervention especially in designing, implementing and sustainability issue. A model of community participation was developed and practiced in child injury prevention project PRECISE (Prevention of Child Injury through Social Intervention) in Bangladesh.

Objective To describe the process of development and role of community groups in injury prevention project in Bangladesh.

Methods Behavioral change issues act as a major factor in injury prevention initiatives so community participation was identified as one of the guiding principle in PRECISE. Village was identified as unit for developing a community group in PRECISE. Prior to develop group 100 people were invited in a meeting; magnitude and consequences of injury and prevention issues were discussed. Discussion also took place how the community people can contribute in injury prevention. After that a 7-10 members Village Injury Prevention committee (VIPC) was formed by the community people. All of the members voluntarily expressed their interest to work for community.

Results 389 VIPC formed and each of the committee has $30 \%$ is female representative. Committee members meet in every month to reviewing present functional status and previous month implementation status of injury prevention initiatives in their village. In the meeting they identify their individual and group responsibilities and set plan of action accordingly. PRECISE has a regular program for the capacity development of the members.

Conclusion PRECISE was found as an effective child injury prevention program in Bangladesh and community participation have a significant contribution in this program.

\section{SP6-63 ANALYSIS OF NEWBORN MORTALITY DUE TO CONGENITAL ANOMALIES ASSOCIATED WITH PERINATAL PERIOD RISK FACTORS}

doi:10.1136/jech.2011.142976q.34

${ }^{1}$ I Zile, ${ }^{*}{ }^{2}$ A Villerusa. ${ }^{1}$ Riga Stradin's University, Public Health and Epidemiology Department, Riga, Latvia; ${ }^{2}$ The Centre of Health Economics, Riga, Latvia

Background Congenital anomalies play a significant role in perinatal mortality. Congenital anomalies are a cause of death in a third of all babies that die in the first year of life in Latvia.

Methods The Register of Newborns was used to identify all babies born 2000-2007 and who were diagnosed with a congenital anomaly (Q00-099). HRs with 95\% CI were estimated using Cox regression model adjusted for mother's age, newborns' gender, different complications during deliveries and pregnancies, gestational time, newborns' birth weight and antenatal care factors.

Results Congenital malformations of the circulatory system were diagnosed in $41.9 \%$ (95\% CI $32.7 \%$ to $51.7 \%$ ) of all babies that died in the first year of life; $13.2 \%$ (95\% CI $7.8 \%$ to $21.2 \%$ ) had musculoskeletal malformations. Multiple anomalies and serious anomalies, such as diaphragmatic hernia and malformations of the digestive system were found in $7.9 \%$ (95\% CI $4.1 \%$ to $14.9 \%$ ). The risk of death in newborns with congenital malformations of the 